\title{
VALORACIÓN ACTITUDINAL FRENTE A TEMAS AMBIENTALES
}

\author{
Marta Fuentealba Cruz ${ }^{1}$ \\ Leonardo Soto Troncoso ${ }^{2}$
}

Recibido el 21 de mayo de 2015, aprobado el 23 de octubre de 2015 y actualizado el 25 de mayo de 2016

DOI: 10.17151/luaz.2016.43.19

\section{RESUMEN}

Se aplicó la Escala de Actitudes de Moreno, Corraliza \& Ruiz (2005) a 367 estudiantes de la ciudad de Talca. Se confirmó una adecuada consistencia interna de la Escala, a Cronbach $=0,776$. Se detectó que en las variables actitudinales, correspondientes al marco personal, es donde se producen las mayores diferencias significativas en sus respuestas, esto es valoración y obligación moral, y las menores diferencias significativas se presentaron en las variables del ámbito contextual: norma social, facilitación e información. Esto es relevante, ya que indica que las variables pertenecientes al ámbito contextual son las que se deben reforzar, lo cual se puede realizar robusteciendo el Currículum a través del fortalecimiento de la educación ambiental. Por otra parte, se concluye que las personas construyen sus actitudes hacia temas emergentes por referencia a sus conocimientos, valores y creencias generales, dando soporte a un modelo de orden jerárquico sobre conocimiento-conciencia-valor-actitud-acción.

\section{PALABRAS CLAVE}

Escala de actitud ambiental, educación ambiental, medio ambiente.

\section{ATTITUDE ASSESSMENT TO ENVIRONMENTAL ISSUES}

\section{ABSTRACT}

The scale of attitudes by Moreno, Corraliza \& Ruiz (2005) was applied to 367 students from the city of Talca. An adequate internal consistency of the scale is confirmed; $\alpha$ Cronbach $=0.776$. It was detected that it is in the attitudinal variables, corresponding to the personal framework, where the major differences occur i.e., assessment and moral obligation, and the minor differences were found in the 
contextual scope variables: social norm, facilitation and information, respectively. This is relevant, since it tells us that the contextual scope variables are the ones that must be strengthened by enhancing the Curriculum through the reinforcing of the environmental education. On the other hand, we believe that people build their attitudes towards emerging issues by making reference to their knowledge, values, and general beliefs, giving support to a hierarchical order model based on knowledge-awareness-value-attitude and action.

\section{KEY WORDS}

Environmental attitude scale, environmental education, environment.

\section{INTRODUCCIÓN}

Los problemas ambientales, si bien no son nuevos, están teniendo una importancia creciente por las consecuencias que es posible observar a simple vista y que provocan deterioro ambiental, como consecuencia de una falta de preocupación por la calidad del medio ambiente (Goleman, 2009).

Frente a la crisis que esto genera se provoca, indudablemente, una necesidad de cambio, que puede ser favorable o desfavorable, la que es posible inducir mediante diversos mecanismos de intervención para crear conciencia y moldear los estilos de vida (Baquedano, 2008). Desde esta perspectiva, la educación como un instrumento de transformación social, es un camino viable para generar cambios favorables frente a los conflictos ambientales, no solo creando conciencia, sino también facilitando el espacio de formación para personas intrínsecamente conscientes de los daños ambientales y de las posibilidades de solucionar problemas al respecto (Strobl, 2005; Pérez, Pérez \& Quijano, 2009; Juyent \& Kong, 2011). En un país como Chile, que sustenta su economía en la extracción de materias primas, esto es fundamental, ya que no es posible vincular desarrollo cultural y económico, sin una conciencia mínima de conservación y protección del entorno en que se vive y que otorga los recursos para subsistir (Zúñiga \& Fuentealba, 2001).

Son muchos y variados los esfuerzos que se han realizado para generar un cambio, profundo y sostenido en el tiempo, en las conductas de las personas para procurar la preservación del ambiente natural. De esta forma, la educación, 
entendida como agente de cambio y transformación social, adquiere un rol preponderante para generar y promover cambios en la esfera social, a través de la sensibilización, concientización y presentación de contenidos ambientales; así, la Educación Ambiental se presenta como una alternativa para generar valores que se traducen en conductas y actitudes favorables para promover el desarrollo equilibrado en armonía con el entorno circundante, desarrollando capacidades plenas en las diversas dimensiones del ser humano, es decir, intelectual, moral y espiritualmente, lo que irremediablemente se refleja en el ámbito actitudinal de las personas (UNESCO, 2004; González-Gaudiano, 2005; CONAMA, 2009; Fuentealba, 2011).

No resulta fácil plantear una investigación en términos de la valoración de actitudes ambientales. Las dificultades nacen del propio concepto de actitud, cuya falta de concreción resulta en un grave impedimento cuando se pretenden construir teorías sobre cambios actitudinales. Así, en los primeros trabajos que analizan el tema, ya se menciona la existencia de un componente previo a la acción en las actitudes, de una disposición a responder ante situaciones concretas. Hoy día la concepción más extendida sobre actitudes identifica otros dos componentes: las cogniciones y las emociones, las cuales motivan y orientan las respuestas (Moser, 2003). Asumimos como propia la definición de actitud de Pérez, Quijano \& Pérez (2005), según la cual "Una actitud es una idea cargada de emotividad que predispone una clase de acciones a una clase particular de situaciones sociales (pág. 29)", ya que incluye numerosas ideas de otros autores y engloba el enfoque particular que ha de considerarse en un sistema formativo como es el educativo. La atención que se debe prestar a las actitudes se justifica al ser consideradas una categoría superior del aprendizaje humano, ya que tienen como referente los valores y las normas, que poseen un carácter relativamente estable y multidimensional y ayudan a ajustarnos a nuestro medio ambiente (Escámez \& Ortega, 1989; González \& Amérigo, 1999; Pérez et al., 2005; Pérez et al., 2009).

Desde hace más de cuatro décadas se vienen desarrollando una multitud de cuestionarios y escalas para contrastar y medir las actitudes hacia el medio ambiente, en general, y hacia diversos problemas ambientales concretos, en particular. Dos clásicos ejemplos de ambos enfoques son las escalas Nuevo Paradigma Ambiental (escala NEP) (Dunlap \& Van Liere, 1978) y Preocupación Ambiental (Weigel \& Weigel, 1978). La primera pretende abarcar la visión de la relación ser humano-naturaleza evaluando el conjunto de creencias que explican cómo funciona el mundo y la biosfera y cómo esta es afectada por las conductas humanas. La segunda, en cambio, examina las actitudes hacia temas ambientales 
específicos como la protección de especies y recursos naturales, la contaminación industrial y la asociada a la energía, el transporte y a la producción y uso de productos de consumo.

Por otra parte, el International Social Survey Program elaboró una encuesta de actitudes hacia el medio ambiente y hacia aspectos concretos como el efecto invernadero, los pesticidas, los residuos urbanos, con objeto de medir la preocupación ambiental en 20 países y obtener un índice de preocupación global (Moreno, Corraliza \& Ruiz, 2005). A su vez, la encuesta Salud del Planeta (Dunlap, Gallup \& Gallup, 1993) fue aplicada en 24 países con tamaños muestréales entre 770 para Finlandia y 5.000 para la India, recopilando un total de 30.000 cuestionarios con información relativa a la preocupación ambiental, incluyendo aspectos de la extinción de especies, la pérdida de bosques, el calentamiento global, la disminución de la capa de ozono y la contaminación atmosférica. Por último, la escala Consciencia de las Consecuencias (Stern, Dietz \& Kalof, 1993), que también evalúa la actitud hacia la extinción de especies y el cambio climático. Estos y otros estudios han detectado el hecho de que la mayoría de las personas manifiestan gran preocupación por el medio ambiente, pero la tendencia general en los datos ha resultado en una visión pesimista de la utilidad de las actitudes como predictores de la conducta ecológica.

Por otro lado, las diversas conductas ambientales son relativamente independientes entre sí por las distintas consecuencias y nivel de implicación personal que suponen, existiendo un conjunto específico de factores que sustenta cada comportamiento (McKenzie-Mohr, Nemiroff, Beers \& Desmarais, 1995; Berger, 1997; Dávila \& Chacón, 2004). Inicialmente se recomendaba medir la actitud general hacia el ambiente como criterio de la conducta ecológica (Weigel, Vernon \& Tognacci, 1974). Si bien Dunlap \& Van Liere (1978) manifestaron la relación entre una acción concreta, el apoyo a regulaciones proambientales, y la escala NEP de actitudes generales, Weigel \& Weigel (1978) demostraron que las medidas generales eran mejores predictores de las conductas generales que de las conductas específicas, hallándose una correlación más fuerte entre la escala de actitudes generales y un índice de comportamiento compuesto de múltiples acciones que con acciones simples.

Varios estudios sugieren que la actitud ambiental debe medirse en relación a temas específicos que optimicen la predicción de comportamientos particulares (Maloney \& Ward, 1973; Van Liere \& Dunlap, 1981; Hines, Hungerford \& Tomera, 1987; Vining \& Ebreo, 1992; Taylor \& Todd, 1995; Dietz, Stern \& Guagnano, 1998; 
González \& Amérigo, 1999; Stern, 2000; Pérez et al., 2009). Por otra parte, Corraliza, Martín, Moreno \& Berenguer (2002) muestran que la conciencia ambiental debe ser objeto de la gestión ambiental misma, y que la implicación de la comunidad en la proambientalidad exige también cambiar las formas de pensar y de sentir sobre el medio ambiente para cambiar las estrategias de acción.

Actualmente en Chile no existen estudios formales publicados que aborden la problemática de la valoración de la conducta actitudinal frente a problemas ambientales, por lo que el objetivo de este estudio es avanzar en la comprensión de la conciencia ecológica y en el conocimiento de los factores que la componen, a través del análisis de variables actitudinales.

\section{METOdOLOGÍA}

\section{Muestra}

Se realizaron 367 encuestas, entre mayo y junio de 2011, incluyendo estudiantes de educación básica (43 alumnos de octavo año), media (209 alumnos de segundo año) y superior (115 alumnos de primer año de universidad), de la ciudad de Talca.

\section{Instrumento}

Se utilizó la Escala de actitudes hacia temas ambientales de Moreno et al. (2005) con modificaciones; la cual consta de 50 ítems donde cada uno aporta una medida específica, relevante y complementaria de la conciencia ambiental. Tal especificidad se obtiene del cruce de 5 dimensiones psicosociales con 10 problemas ambientales, de modo que cada ítem contiene una combinación única de un aspecto actitudinal y otro ambiental. Las respuestas se miden solicitando el grado de acuerdo para cada ítem en un formato tipo Likert de 4 puntos: (1) nada o casi nada, (2) algo, (3) bastante y (4) mucho o totalmente (Cañadas \& Sánchez, 1998; Ospina, Sandoval, Aristizábal \& Ramírez, 2005). Tal como se recomienda en la bibliografía, la mitad de las proposiciones de la escala se presentaron de manera positiva y el resto de forma negativa (Pliego, Contini, Odetti, Güemes \& Tiburzi, 2004; Hernández \& Jiménez, 2010).

Las variables actitudinales, dentro del ámbito contextual del individuo, incluyen la facilitación de la conducta proambiental, la información y la norma social, y en el 
marco personal, la obligación moral o norma personal y la valoración. La facilitación considera aquellas condiciones externas que facilitan, restringen o inhiben una acción proambiental determinada. Los ítems de información contienen afirmaciones de consenso científico que pretenden medir el conocimiento de un problema ambiental dado. La norma social hace referencia a las reglas percibidas por los individuos de la comunidad. La norma personal indica el sentimiento de obligación para asumir cierta responsabilidad ante los problemas ambientales. Por último, la valoración se basa en el juicio que el individuo realiza sobre la seriedad del problema (Tabla 1).

Tabla 1. İtems por variable actitudinal, de acuerdo a Moreno et al. (2005)

\begin{tabular}{|l|l|}
\hline Variable actitudinal & Items de la Escala de actitudes hacia problemas ambientales \\
\hline Valoración & $47-50-3-29-37-31-1-44-12-27$ \\
\hline Facilitación & $43-49-36-41-28-13-48-18-22-11$ \\
\hline Norma social & $20-15-35-23-45-5-10-7-2-46$ \\
\hline Obligación moral & $32-25-21-17-24-40-26-34-4-39$ \\
\hline Información & $38-14-19-42-33-6-30-16-9-8$ \\
\hline
\end{tabular}

Las variables ambientales incluyen 10 tipos de problemas que recogen los principales contenidos de la crisis ambiental. Un bloque sobre contaminación integra problemas de ámbito regional y global como los productos químicos o el efecto invernadero y de ámbito local como el transporte, las basuras y el ruido en las ciudades. El bloque de conservación aborda el uso y estado de recursos como la energía y el agua, la conservación de espacios naturales, biodiversidad y reciclaje (Tabla 2). 
Tabla 2. Items por variables ambientales, de acuerdo a Moreno et al. (2005)

\begin{tabular}{|l|l|}
\hline Variable ambiental & Items de la Escala de actitudes hacia problemas ambientales \\
\hline Espacios naturales & $31-43-45-25-33$ \\
\hline Contaminación & $39-14-10-44-49$ \\
\hline Basuras & $40-6-20-50-36$ \\
\hline Energía & $41-1-35-42-34$ \\
\hline Agua & $37-28-23-4-8$ \\
\hline Biodiversidad & $47-13-7-17-30$ \\
\hline Reciclaje & $29-48-15-32-38$ \\
\hline Químicos & $26-16-5-18-3$ \\
\hline Transporte & $12-22-2-24-9$ \\
\hline Ruido & $27-11-46-21-19$ \\
\hline
\end{tabular}

\section{Análisis de los datos}

El análisis de los resultados de la escala se realizó con estadística descriptiva y multivariada, para lo cual se utilizó el software Statistical Package for Social Science versión 15.0 .

\section{RESULTADOS}

El coeficiente alfa de Cronbach que mide la fiabilidad de la escala de medición fue de 0,776 , lo que demostró que el instrumento de medición es fiable.

Aunque la población encuestada conocía de manera general los problemas ambientales, se determinó que tenía mayor información sobre los temas de contaminación, energía, residuos sólidos urbanos, espacios naturales y menor información sobre ruido y transporte; quedando en posición intermedia los conocimientos sobre agua, reciclaje, biodiversidad y químicos, como se aprecia en la Figura 1, que grafica las variables ambientales de la escala como porcentajes de la suma de las categorías "bastante" y "mucho o totalmente". Además, se puede observar que los porcentajes más altos de estas categorías correspondieron a estudiantes de nivel superior. 
Al clasificar las variables actitudinales de la escala como los porcentajes de la suma de las categoría "bastante" y "mucho o totalmente" se detectó que las correspondientes al marco personal (valoración y obligación moral) son las que alcanzaron los más altos porcentajes de respuestas, tanto en educación superior, media y básica (Figura 2).

En la Tabla 3 se muestran los resultados del análisis de varianza considerando como variable independiente el nivel de estudio y como variables dependientes las puntuaciones obtenidas de las diferentes variables ambientales y actitudinales en estudio. Se obtuvieron diferencias estadísticas significativas en las variables pertenecientes a los aspectos de valoración, obligación moral, información y facilitación. Sin embargo, no se detectaron diferencias estadísticas significativas en aspecto de norma social entre los grupos en estudio.
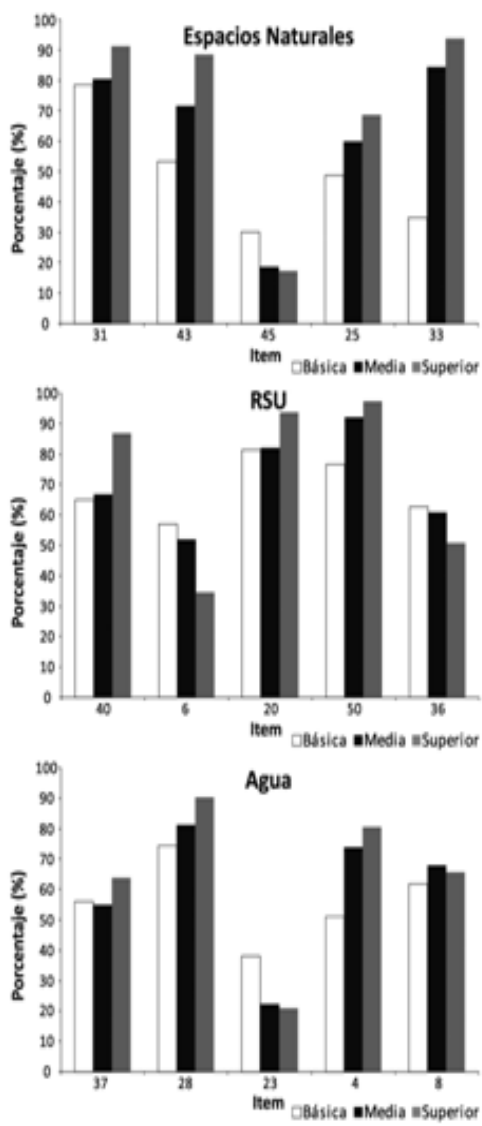
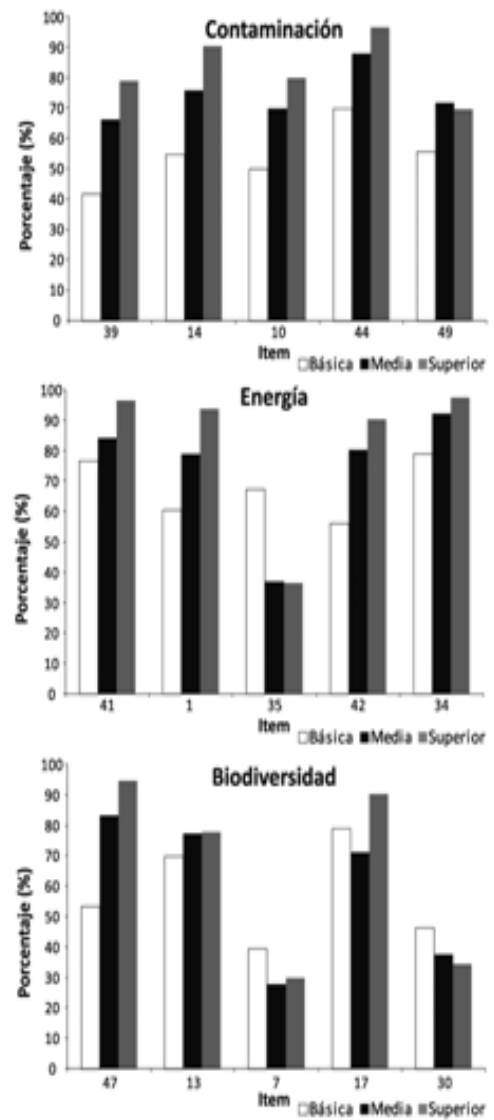

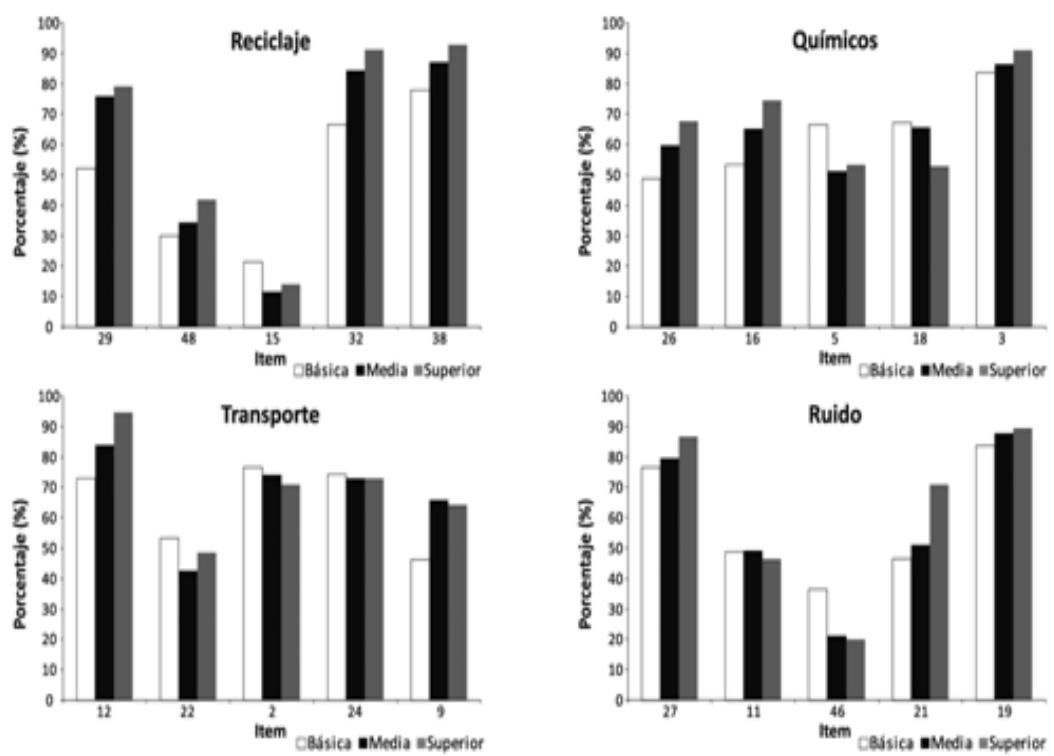

Fuente: los autores.

Figura 1. Histograma de las variables ambientales como porcentajes de la suma de las categorías "bastante" y "mucho o totalmente".
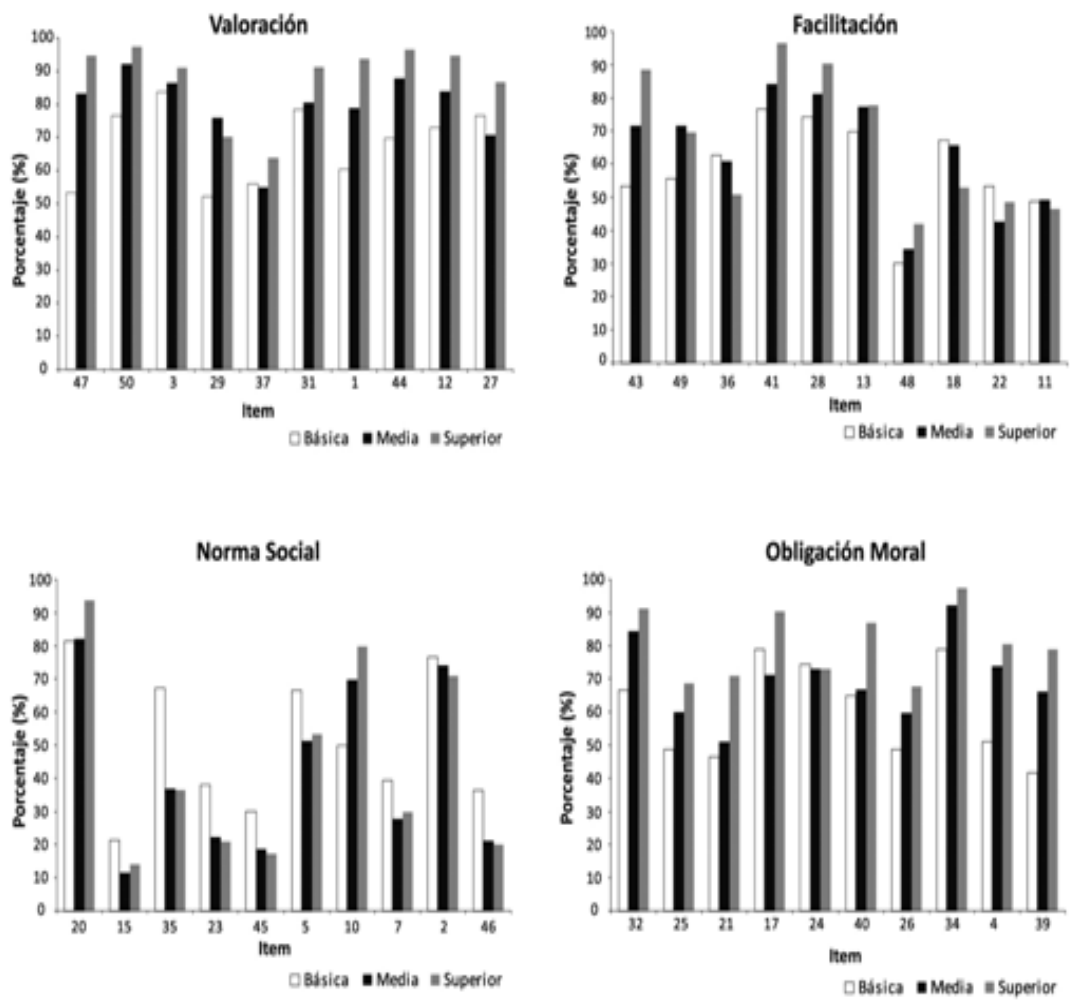


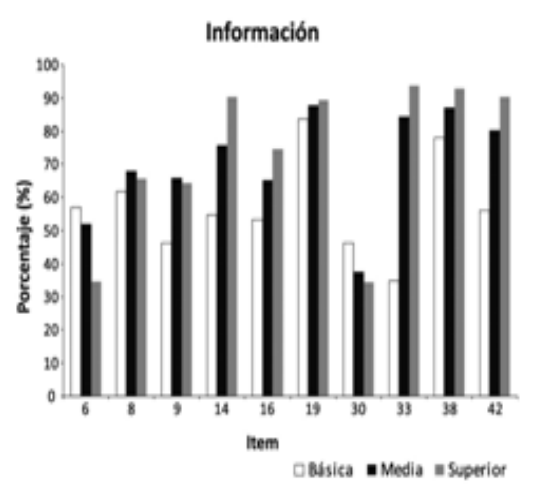

Fuente: los autores.

Figura 2. Histograma de las variables actitudinales como porcentajes de la suma de las categorías "bastante" y "mucho o totalmente".

Tabla 3. Análisis de varianza entre los grupos de estudio

\begin{tabular}{|c|c|c|c|c|c|c|c|c|c|}
\hline \multicolumn{2}{|c|}{ Valoración } & \multicolumn{2}{|c|}{ Facilitación } & \multicolumn{2}{|c|}{ Norma social } & \multicolumn{2}{|c|}{ Obligación moral } & \multicolumn{2}{|c|}{ Información } \\
\hline item & $F$ & item & $F$ & item & $\bar{F}$ & item & $F$ & item & $F$ \\
\hline 47 & $22,136^{\text {***8 }}$ & 43 & $15,409 * * 2$ & 20 & 4,956 & 32 & $10,352^{* * *}$ & 6 & 6,361 \\
\hline 50 & $12,065^{\text {* }}$ & 49 & 1,661 & 15 & 0,208 & 25 & 1,670 & 8 & 0,969 \\
\hline 3 & 0,420 & 36 & 0,458 & 35 & 6,716 & 21 & 6,900 & 9 & 3,202 \\
\hline 29 & 6,164 & 41 & $15,281^{\text {*** }}$ & 23 & 4,021 & 17 & 5,341 & 14 & $12,650^{* * *}$ \\
\hline 37 & 5,722 & 28 & 6,905 & 45 & 1,883 & 24 & 0,941 & 16 & 4,109 \\
\hline 31 & 4,690 & 13 & 1,199 & 5 & 2,229 & 40 & $12,413^{\mathrm{k} * \mathrm{z}}$ & 19 & 3,129 \\
\hline 1 & 9,112 & 48 & 0,884 & 10 & 6,773 & 26 & 3,850 & 30 & 0,597 \\
\hline 44 & 13,869 *** & 18 & 0,776 & 7 & 2,555 & 34 & $17,774^{* * *}$ & 33 & $58,282^{* * 2 * 8}$ \\
\hline 12 & 11,976 *** & 22 & 1,106 & 2 & 0,515 & 4 & 8,867 *** & 38 & 7,230 \\
\hline 27 & 1,175 & 11 & 0,264 & 46 & 2,860 & 39 & $7,972^{* * *}$ & 42 & $14,553^{\text {*** }}$ \\
\hline
\end{tabular}

$* * *<0,0001$.

g.l. $=366$.

Se realizó un análisis de componentes principales (PCA) con la finalidad de detectar posibles diferencias entre estudiantes de diversos niveles educacionales (básico, medio y superior). LaTabla 4 resume los autovalores de las funciones canónicas discriminantes del PCA, donde pueden observarse las desviaciones de las puntuaciones discriminantes entre grupos respecto a las desviaciones dentro de los grupos; puede destacarse que la función 1 concentra el $70,6 \%$ de la variabilidad. Se observa que la lambda de Wilks es significativa en las dos primeras funciones $p<0,0001$ (Tabla 5). 
Tabla 4. Resumen de funciones canónicas discriminantes

\begin{tabular}{|c|c|c|c|c|}
\hline Función & Autovalor & \% de varianza & \% acumulado & Correlación canónica \\
\hline 1 & 0,825 & 70,6 & 70,6 & 0,672 \\
\hline 2 & 0,344 & 29,4 & 100,0 & 0,506 \\
\hline
\end{tabular}

Fuente: los autores.

Tabla 5. Lambda de Wilks

\begin{tabular}{|c|c|c|c|c|}
\hline Contraste de las funciones & Lambda de Wilks & Chi-cuadrado & gl & Sig \\
\hline 1 a la 2 & 0,408 & 206,729 & 100 & 0,000 \\
\hline 2 & 0,744 & 68,103 & 49 & 0,037 \\
\hline
\end{tabular}

Fuente: los autores.

La Figura 3 entrega una proyección de los resultados sobre el plano definido por las dos primeras funciones, donde es posible observar que el centroide del grupo 1 (básico) está en sector negativo de la función 1 y en el sector positivo de la función 2 , distante de los centroides de los grupos 2 (media) y 3 (superior) que están en el sector negativo y en el sector positivo de ambas funciones respectivamente (Tabla $6)$. 


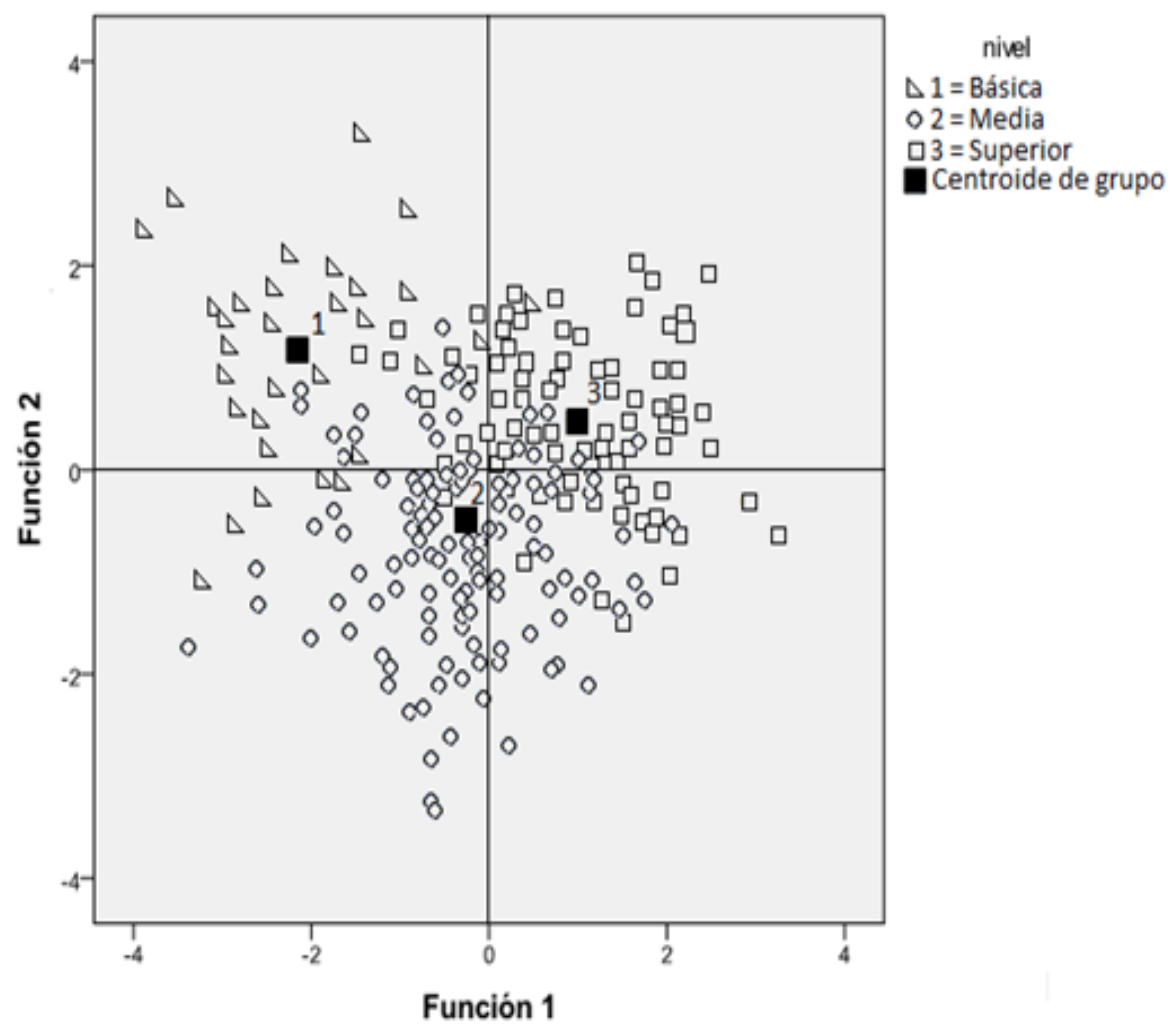

Fuente: los autores.

Figura 3. Proyección de resultados de los estudiantes encuestados sobre el plano definido por las dos primeras funciones del PCA.

Tabla 6. Funciones en los centroides de los grupos

\begin{tabular}{|c|c|c|}
\hline \multirow{2}{*}{ Nivel } & \multicolumn{2}{|c|}{ Función } \\
\cline { 2 - 3 } & $\mathbf{1}$ & $\mathbf{2}$ \\
\hline 1 (Básica) & $-2,156$ & 1,173 \\
\hline 2 (Media) & $-0,262$ & $-0,490$ \\
\hline 3 (Superior) & 0,994 & 0,472 \\
\hline
\end{tabular}

Fuente: los autores.

Es importante destacar que el $75,5 \%$ de los casos fue correctamente clasificado en su grupo de pertenencia; siendo el grupo 2 (media) el cual presentó menor porcentaje de pertinencia con un $66,7 \%$, con un $22,2 \%$ de superposición con el 
grupo 3 (superior); de igual forma, el grupo 3 con un $84,4 \%$ de pertinencia presentó un $12,0 \%$ de superposición con el grupo 2 (Tabla 7 ).

Tabla 7. Resultados de la clasificación del PCA (1 = básica, 2 = media, 3 = superior)

\begin{tabular}{|r|c|c|c|c|}
\hline Nivel & \multicolumn{5}{|c|}{ Grupo de pertenencia } \\
\hline & $\mathbf{1}$ & $\mathbf{2}$ & $\mathbf{3}$ & Total \\
\hline Recuento & \multicolumn{4}{|c|}{} \\
\hline 1 & 22 & 0 & 2 & 24 \\
\hline 2 & 16 & 96 & 32 & 144 \\
\hline 3 & 3 & 11 & 76 & 90 \\
\hline Porcentaje & \multicolumn{5}{|c|}{} \\
\hline 1 & 91,7 & 0,0 & 8,3 & 100,0 \\
\hline 2 & 11,1 & 66,7 & 22,2 & 100,0 \\
\hline 3 & 3,3 & 12,0 & 84,4 & 100,0 \\
\hline
\end{tabular}

Clasificados correctamente el $75,2 \%$ de los cas os agrupados originales.

Fuente: los autores.

\section{DISCUSIÓN}

Si bien la literatura muestra interés por el estudio a nivel específico de las actitudes ambientales (Castañedo, 1995; Manassero \& Vázquez, 2001; Moreno et al., 2005; Manzanal, Rodríguez \& Zamora, 2006; Olave de Amigo, 2006; Álvarez \& Vega, 2009; Rivera-Jacinto \& Rodríguez-Ulloa, 2009; Hernández \& Jiménez, 2010; Vargas, Vázquez, Gutiérrez, Vargas \& Fernández, 2010; Vargas-Mendoza, Maldonado-Aragón, Cruz-Clemente \& Aguilar-Morales, 2012) en Chile no existen reportes al respecto. Tomando en cuenta lo anterior, este trabajo es un aporte a la comprensión de la conciencia ecológica que muestran los jóvenes; la cual debería considerarse al momento de diseñar programas de protección ambiental, y para incluir en los programas educativos de nivel superior temas relacionados con la educación ambiental, como respuesta a la responsabilidad de formadores de profesionales íntegros con alta valoración por el medio ambiente y protección a los recursos naturales (Sosa, Isaac-Márquez, Eastmond, Ayala \& Arteaga, 2010; Vargas et al., 2010; Vargas, Moreno, Vázquez \& Gutiérrez, 2011). 
Se han diseñado diferentes escalas de actitudes específicas hacia temas ambientales concretos, como aproximación integradora de la crisis ecológica en ambos aspectos: psicológico y ambiental. Estos han sido aplicados en diferentes lugares, obteniéndose como resultado una alta variabilidad de consistencia interna. Así por ejemplo, Moreno et al. (2005) señalaron que la consistencia interna del índice de preocupación global del ISSP varió desde 0,23 en Filipinas a 0,72 en Alemania occidental; por su parte, Moreno et al. (2005) obtuvieron una consistencia interna de 0,83 para su escala. En el presente estudio se obtuvo una consistencia interna de 0,77 para la escala modificada de Moreno et al. (2005), por lo que se puede concluir que su uso es recomendable para contrastar la evolución de la conciencia ambiental.

Es importante destacar que las mayores diferencias se produjeron en las variables actitudinales correspondientes al marco personal, que incluyen valoración y obligación moral, y las menores diferencias se presentaron en las variables del ámbito contextual que incluyen las normas sociales, facilitación e información. Esto es relevante, ya que indica que las variables pertenecientes al ámbito contextual son las que se deben reforzar, lo cual se puede realizar robusteciendo el Currículum a través del fortalecimiento de la educación ambiental.

En general, los factores denominados contextuales o estructurales hacen referencia a todo un conjunto de variables, incluyendo las sociodemográficas, tecnológicas, geográficas, sociales, económicas y políticas, las físicas o inhibidoras y también las leyes, normativas o regulaciones institucionales y políticas públicas (Álvarez \& Vega, 2009). Al incluir estas variables en las estrategias educativas diseñadas, se producirá un aumento del conocimiento sobre el medio ambiente, que normalmente tienen una importante influencia en la conducta ambiental individual y también en la conducta ambiental de las organizaciones (Stern, 2000; Sosa et al., 2010).

Por su parte, Dietz et al. (1998) señalaron que las variables de contexto tendrían una influencia directa sobre la conducta actuando ya sea como facilitadores o como inhibidores de su puesta en marcha y también tendrían un efecto indirecto a través de las influencias socioculturales que configuran los procesos cognitivos. A este respecto, es posible que los sistemas de valores compartidos y los patrones de relaciones sociales e interpersonales influyan positivamente en las percepciones y estrategias que los individuos mantienen sobre la interacción con el medio ambiente. 
Por lo tanto, está claro que las variables contextuales, en su más amplio sentido, tienen un peso determinante sobre la preocupación y conducta ecológica; lo cual está directamente relacionado con los valores humanos. A través de constructos cognitivos se activan o construyen normas personales o sentimientos de obligación moral, en forma de reglas de comportamiento con las que se evalúan los hechos y se decide lo que hay que hacer en una determinada situación (Corral-Verdugo \& De Quiroz, 2004; Manzanal et al., 2006; Álvarez \& Vega, 2009; Fuentealba, Roco, Marín \& Castillo, 2015). De esta forma, los valores y las creencias funcionarían como guías o heurísticos que activan o generan actitudes o normas de acción específicas ante una situación ambiental particular.

De aquí, surge la importancia del fortalecimiento de la educación ambiental en la educación formal, tanto en la capacitación del profesorado como de los estudiantes (Espejel \& Castillo, 2008; Vargas et al., 2011; Fuentealba et al., 2015). Queda claro que el conocimiento sobre el medio ambiente ayudaría a formar conciencia del impacto de las actividades antrópicas sobre los ecosistemas y sus efectos sobre el bienestar humano. A su vez, la conciencia ambiental conlleva a valorar los diferentes ecosistemas, lo que debería conducir a la adopción de una actitud y toma de acciones ecológicas. De esta forma, a través de la educación ambiental correctamente dirigida, las personas pueden construir sus actitudes hacia temas emergentes por referencia a sus conocimientos, valores y creencias generales, dando soporte a un modelo de orden jerárquico sobre conocimiento-concienciavalor-actitud-acción.

\section{REFERENCIAS}

- Álvarez, P., \& Vega, P. (2009). Actitudes ambientales y conductas sostenibles, implicaciones para la educación ambiental. Revista de Psicodidáctica, 14(2), 245-260.

- Baquedano, M. (2008). Tu huella ecológica. Santiago: Cuatro Vientos.

- Berger, I. (1997). The demographics of recycling and the structure of environmental behavior.Environment and Behavior, 29(4), 515-531.

- Cañadas, I., \& Sánchez, A. (1998). Categorías de respuesta en escalas tipo likert.Psicotherma, 10(3), 623-631. 
- Castañedo, C. (1995). Escala para la evaluación de las actitudes proambientales. Revista Complutense de Educación, 6(2), 253-278

- CONAMA. (2009). Política Nacional de Educación para el Desarrollo Sustentable. Santiago: CONAMA.

- Corral-Verdugo, V., \& De Queiroz, J. (2004). Aproximaciones al estudio de la conducta sustentable. Medio Ambiente y Comportamiento Humano, 5(1), 1-26.

- Corraliza, J., Martín, R., Moreno, M., \& Berenguer, J. (2002). El estudio de la conciencia ambiental. Revista Medio Ambiente, 21(40), 36-38.

- Dávila, M. C., \& Chacón, F. (2004). Factores psicosociales y tipo de voluntariado.Psicothema, 16(4), 639-645.

- Dietz, T., Stern, P., \& Guagnano, G. (1998). Social structural and social psychological bases of environmental concern. Environment and Behavior, 30(4), 450-471.

- Dunlap, R., Gallup, G., \& Gallup, A. (1993). Of global concern. Results of the health of the Planet Survey. Environment: Science and Policy for Sustainable Development, 35(9), 6-22.

- Dunlap, R., \& Van Liere, K. (1978). The "new environmental paradigm": a proposed instrument and preliminary results. Journal of Environmental Education, 9(4), 10-19.

- Escámez, J., \& Ortega, P. (1989). La comunicación en la educación moral y el cambio de actitudes. En Conceptos y Propuestas. Vol. V. Papers d’Educació. Valencia: Nau Llibres.

- Espejel, A., \& Castillo, M. (2008). Educación ambiental para el nivel medio superior: propuesta y evaluación. Revista Iberoamericana de Educación, 46(2), $1-11$.

- Fuentealba, M. (2011). Reflexión sobre impacto ambiental antrópico, desarrollo sustentable y educación ambiental. UCMaule, 41, 29-43.

- Fuentealba, M., Roco, L., Marín, F., \& Castillo, F. (2015). Reflexiones pedagógicas en torno al impacto al campamento EXLORA CHILE VA! Valorando la 
biodiversidad maulina del la UCM. En 3er Encuentro Nacional de Divulgadores de Ciencia y Tecnología (pp. 343-350).

- Goleman, D. (2009). Inteligencia ecológica. Madrid: Kairós

- González, A., \& Amérigo, M. (1999). Actitudes hacia el medio ambiente y conducta ecológica. Psicothema, 11(1), 13-25.

- González-Gaudiano, E. (2005). The Role Environmental Education Might Play in the Decade for Education for Sustainable Development. Applied Environmental Education and Communication, 4(3), 203-206.

- Hernández, L., \& Jiménez, G. (2010). Actitudes y comportamiento ambiental del personal del área de conservación marina Isla del Coco, Costa Rica. Biocenosis, 23(1), 2-13.

- Hines, J., Hungerford, H., \& Tomera, A. (1987). Analysis and synthesis of research on responsible environmental behavior: a meta-analysis. Journal of Environmental Education, 18(2), 1-8.

- Juyent, M., \& Kong, F. (2011). Envisioning the future: a case study in a primary school in Barcelona. Eingana, 34(3), 16-23.

- Maloney, M., \& Ward, M. (1973). Ecology: let's hear from the people. An objective scale for the measurement of ecological attitudes and knowledge. American Psychologist, 28(7), 583-586.

- Manassero, M., \& Vázquez, A. (2001). Instrumentos y métodos para la evaluación de las actitudes relacionadas con la ciencia, la tecnología y la sociedad. Enseñanza de la Ciencias, 20(1), 15-27.

- Manzanal, R., Rodríguez, L., \& Zamora, J. (2006). El conocimiento de las actitudes ambientales: una buena base para mejorar las conductas hacia el medio ambiente. En III Jornadas de Educación Ambiental de la Comunidad Autónoma de Aragón (pp. 1-7). CIAMA, La Alfranca, Zaragoza.

- McKenzie-Mohr, D., Nemiroff, L., Beers, L., \& Desmarais, S. (1995). Determinants of responsible environmental behavior. Journal of Social Issues, 51(4), 139-156.

- Moreno, M., Corraliza, J., \& Ruiz, J. (2005). Escala de actitudes ambientales hacia problemas específicos. Psicothema, 17(3), 502-508. 
- Moser, G. (2003). Environmental psychology for the 21st century: The challenge of sustainable development. Revista de Psicología de la Universidad de Chile, 12(2), 11-17.

- Olave de Amigo, R. (2006). Conocimientos y actitudes ante el ambiente para la incorporación de la dimensión ambiental en el pensum de ingeniería industrial. Universidad, Ciencia y Tecnología, 10(38), 62-66. ISSN 1316-4821.

- Ospina, B., Sandoval, J., Aristizábal, C., \& Ramírez, M. (2005). La escala de Likert en la valoración de los conocimientos y las actitudes de los profesionales de enfermería en el cuidado de la salud. Antioquia. 2003. Investigación y Educación en Enfermería, 23(1), 14-29.

- Pérez, M. Á, Pérez, M., \& Quijano, R. (2009). Valoración del cambio de actitudes hacia el medio ambiente producido por el programa didáctico "EICEA" en los alumnos de Educación Secundaria Obligatoria (14-16 años). Revista Electrónica de Enseñanza de las Ciencias, 8(3), 1019-1036.

- Pérez, M., Quijano, R., \& Pérez, M. (2005). Actitudes del profesorado de Educación Secundaria Obligatoria, respecto al desarrollo de capacidades profesionales básicas. Málaga: Aljibe.

- Pliego, O., Contini, L., Odetti, H., Güemes, R., \& Tiburzi, M. (2004). Las actitudes de los estudiantes universitarios hacia el fenómeno radiactivo, la energía nuclear y sus aplicaciones.Educación Química, 15(2), 142-148.

- Rivera-Jacinto, M., \& Rodríguez-Ulloa, C. (2009). Actitudes y comportamientos ambientales en estudiantes de enfermería de una Universidad Pública del Norte de Perú. Rev. Perú Med. Exp. Salud Pública, 26(3), 338-342.

- Sosa, S., Isaac-Márquez, R., Eastmond, A., Ayala, M., \& Arteaga, M. (2010). Educación superior y cultura ambiental en el sureste de México. Universidad y Ciencia, 26(1), 33-49.

- Stern, P. (2000). Toward a coherent theory of environmentally significant behavior. Journal of Social Issues, 56(3), 407-424.

- Stern, P., Dietz, T., \& Kalof, L. (1993). Value orientations, gender and environmental concern.Environment and Behavior, 25(5), 322-348. 
- Strobl, G. (2005). Una Educación Ambiental Conforme a la Época. Revista de Educación Ambiental, 5, 48-50.

- Taylor, S., \& Todd, P. (1995). An integrated model of waste management behavior. A test of household recycling and composting intentions. Environment and Behavior, 27(5), 603-630.

- UNESCO. (2004). Década de la Educación para el Desarrollo Sostenible (20052014).París.

- Van Liere, K., \& Dunlap, R. (1981). Environmental concern: does it make a difference how it's measured? Environment and Behavior, 13(6), 651-676.

- Vargas, C., Moreno, J., Vázquez, L., \& Gutiérrez, G. (2011). Actitudes ambientales en los estudiantes de nivel superior en México. Luna Azul, 33, 31-36. Recuperado de http://www.scielo.org.co/pdf/luaz/n33/n33a04.pdf

- Vargas, C., Vázquez, L., Gutiérrez, G., Vargas, A., \& Fernández, C. (2010). Conciencia ambiental en estudiantes de Enfermería Básica. Revista Universitaria de Investigación y Diálogos Académicos, 6(3), 18-28.

- Vargas-Mendoza, J., Maldonado-Aragón, M., Cruz-Clemente, M., \& AguilarMorales, J. (2012). Environmental attitudes and behaviors in psychology and architecture students in Oaxaca city, Mexico. Centro Regional de Investigación en Psicología, 6(1), 7-12.

- Vining, J., \& Ebreo, A. (1992). Predicting recycling behavior from global and specific environmental attitudes and changes in recycling opportunities. Journal of Applied Social Psychology, 22(20), 1580-1607.

- Weigel, R., Vernon, D., \& Tognacci, L. (1974). Specificity of the attitude as a determinant of attitude-behavior congruence. Journal of Personality and Social Psychology, 30(6), 724-728.

- Weigel, R., \& Weigel, J. (1978). Environmental concern. The development of a measure.Environment and Behavior, 10(1), 3-15.

- Zúñiga, M., \& Fuentealba, M. (2001). Sustentabilidad, ecología industrial y capitalismo natural. UCMaule, 27, 7-16. 
1. Doctora en Ciencias Ambientales. Universidad Católica del Maule, Facultad de Ciencias Básicas. Talca. Chile. mfuentea@ucm.cl. ORCID: 0000-0003-2135-6869 2. Sociólogo. Fondo Solidario de Inversión Social (FOSIS). Talca. Chile.leonardo.soto@fosis.gob.cl. ORCID: 0000-0002-8417-4459

Para citar este artículo: Fuentealba-Cruz, M., \& Soto-Troncoso, L. (2016). Valoración actitudinal frente a temas ambientales. Revista Luna Azul, 43, 448467.

Recuperado de

http://200.21.104.25/lunazul/index.php?option=com_content\&view=article\&id=208

Esta obra está bajo una Licencia de Creative Commons Reconocimiento CC BY

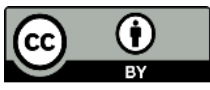

